\title{
Mechanical behavior of the erythrocyte in microvessel stenosis
}

\author{
ZHANG ZhiGuo ${ }^{1,2} \&$ ZHANG XiWen ${ }^{2 *}$ \\ ${ }^{1}$ State Key Laboratory of Structural Analysis for Industrial Equipment, Dalian University of Technology, Dalian 116024, China; \\ ${ }^{2}$ School of Aerospace, Tsinghua University, Beijing 100084, China
}

Received August 21, 2009; accepted February 22, 2010; published online March 17, 2011

\begin{abstract}
The passage of red blood cells (RBCs) through capillaries is essential for human blood microcirculation. This study used a moving mesh technology that incorporated leader-follower pairs to simulate the fluid-structure and structure-structure interactions between the RBC and a microvessel stenosis. The numerical model consisted of plasma, cytoplasm, the erythrocyte membrane, and the microvessel stenosis. Computational results showed that the rheology of the RBC is affected by the Reynolds number of the plasma flow as well as the surface-to-volume ratio of the erythrocyte. At a constant inlet flow rate, an increased plasma viscosity will improve the transit of the RBC through the microvessel stenosis. For the above reasons, we consider that the decreased hemorheology in microvessels in a pathological state may primarily be attributed to an increase in the number of white blood cells. This leads to the aggregation of RBCs and a change in the blood flow structure. The present fundamental study of hemorheology aimed at providing theoretical guidelines for clinical hemorheology.
\end{abstract}

red blood cell, moving mesh, leader-follower pair, rheology, fluid-structure interaction

Citation: Zhang Z G, Zhang X W. Mechanical behavior of the erythrocyte in microvessel stenosis. Sci China Life Sci, 2011, 54: 450-458, doi: $10.1007 / \mathrm{s} 11427-011-4152-3$

It is well known that the hematocrit in human arteries is about $40 \%$ and that this value is lower in microvessels [1,2]. Red blood cells (RBCs) carry oxygen from the lungs to tissues and nutrient substances to organs, while removing carbon dioxide and waste products. Hence, the biomechanical behavior of RBCs in microcirculation is very important [3,4]. Many experiments and numerical simulations have been performed over the past several decades to further our understanding of the biomechanical behavior of RBCs [5-26].

The RBC is composed of liquid cytoplasm, and the cell membrane, which has viscoelastic properties. In general, it is believed that the degree of deformability of the RBC is controlled by the flexibility of the cell membrane. Therefore, numerous measurements and simulations on the rheology of the cell membrane have been performed on populations of RBCs, as well as at the single-cell level. Techniques for the

*Corresponding author (email: zhangxiw@tsinghua.edu.cn) measurement of single cells have included micropipette aspiration [5], optical tweezers [6,7] and high-frequency electrical deformation tests [8], in which RBCs are subjected to large deformations and static loads. Knowledge of cell membrane mechanics contributes to the understanding of RBC collective behaviors in tissues and organs.

The deformability of RBCs affects blood rheology, cell structure, and movement through the capillaries; hence the overall microcirculation. In light of the complexity of cell motion and deformation in microvessels, simulations on the motion and deformation of RBCs under flow in microvessels could serve as a valuable tool for shear stress measurements and for quantifying the biomechanical parameters of RBCs. Fung [9], Skalak [10,11] and Evans et al. [5] have performed a number of theoretical studies on relatively simple axisymmetric models of RBC in capillaries. The deformability of the RBC, in theoretical studies, has been shown to have a direct relationship with its mechanical properties. The biconcave shape of the RBC enables it to 
deform into a wide variety of shapes. A number of increasingly comprehensive models that combine cell deformation, fluid dynamics, and membrane mechanics have been proposed and various numerical methods have been utilized, including the boundary element method [12-15], the finite element method [16-18], the immersed boundary method [19,20] and the perturbation method [21,22]. Recently, Toru et al. [23] used the lattice Boltzmann method (LBM) to study the flow of RBCs with liposome-encapsulated hemoglobin at microvascular bifurcations. So far, all of these studies have focused only on RBCs flowing in the normal microvascular vessels, as shown in Figure 1A. To the best of our knowledge, few studies have focused on the mechanical behavior of RBCs transiting through a microvessel stenosis, which may provide us with a less ambiguous picture of the deformation of RBCs and a more convenient method to estimate the rheological properties of human microcirculation. The present study specifically investigates the movement of a single RBC in a microvessel with a moderate stenosis, as shown in Figure 1B.

The deformability of RBCs is an important factor that regulates blood rheology [24-26]. Deformability is the combined result of several mechanical and geometrical properties of the RBC, such as the viscosity ratio between cytoplasm and plasma, the surface-to-volume ratio of the cells, the Reynolds number of the blood flow, and the material coefficients of the cell membrane. The ability of a cell to change its shape determines whether or not that cell can pass through small vessels, especially capillaries with stenoses, which is a physiologically important aspect of RBC deformability. The present study uses a moving mesh technology that incorporates a finite element method to investigate the biomechanical behavior of a single RBC transiting through a microvessel stenosis. Our purpose is to provide theoretical guidelines for clinical hemorheology.

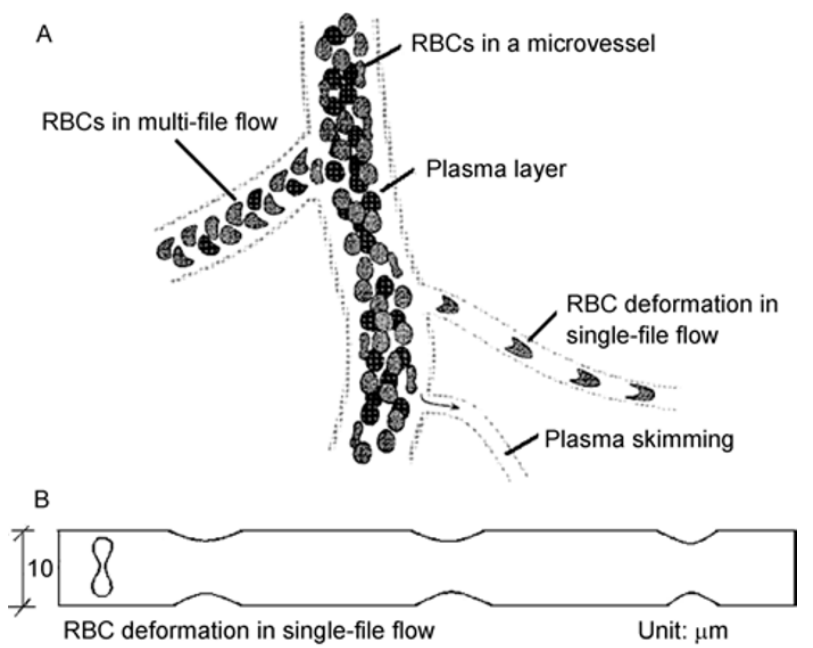

Figure 1 A schematic of the RBCs flowing in human microvessels (A) and the single-file flow of RBCs in a microvessel with moderate stenosis (B).

\section{Materials and methods}

\subsection{Assumptions}

Figure 2A shows a schematic of the axisymmetric model used to investigate the motion of a single RBC passing through a microvessel stenosis. The diameter and length of the microvessel are 10 and $158 \mu \mathrm{m}$, respectively. The RBC is considered to be a biconcave discoid with a radial diameter of $8 \mu \mathrm{m}$ and an axial diameter of $1 \mu \mathrm{m}$.

(i) The blood vessel wall was assumed to be rigid and the plasma was assumed to be a homogeneous, incompressible, and Newtonian fluid with viscosity $\mu=1.2 \mathrm{mPa}$ s and density $\rho=1050 \mathrm{~kg} \mathrm{~m}^{-3}$.

(ii) The RBC membrane was assumed to be viscoelastic with a thickness of $0.1 \mu \mathrm{m}$.

(iii) The fluid-structure interactions among cell membrane, cytoplasm, and plasma were studied.

(iv) To investigate the deformability of the RBC, a contact pair between the cell membrane and the microvessel stenosis was established.

(v) Because the cell membrane endures a large deformation, the ALE (Arbitrary Lagrange-Euler) mesh was used to simulate the movement of the fluid region.

\subsection{Theoretical model}

\subsubsection{Governing equations}

The numerical simulation was based on the incompressible and viscous Navier-Stokes equations under steady state conditions. Given the appropriate dimensionless variables, the flow can be described in axisymmetric cylindrical coordinates $(x, y)$ by the following equations:

$$
\begin{gathered}
\frac{\partial^{2} \psi}{\partial x^{2}}-\frac{1}{y} \frac{\partial \psi}{\partial y}+\frac{\partial^{2} \psi}{\partial y^{2}}=y \eta \\
\frac{\partial u \eta}{\partial x}+\frac{\partial v \eta}{\partial y}=\frac{2}{\operatorname{Re}}\left[\frac{\partial^{2} \eta}{\partial x^{2}}+\frac{1}{y} \frac{\partial}{\partial y}\left(y \frac{\partial \eta}{\partial y}\right)-\frac{\eta}{y^{2}}\right]
\end{gathered}
$$

where $\psi$ and $\eta$ represent the normalized stream function and the vorticity, respectively. Re represents the Reynolds number of the fluid flow.

\subsubsection{Boundary conditions}

Figure 2B shows the moving mesh and the boundary conditions utilized in the numerical simulation. The boundary conditions on the flow domain were as follows: (i) $\bar{U}=$ $10 \mu \mathrm{m} \mathrm{s}^{-1}$, at the inlet [27]; (ii) $P=0 \mathrm{~Pa}$, at the outlet; (iii) no-slip conditions at the vessel wall.

The solid and fluid domains were coupled together by a direct two-way coupling formulation, whereby the fluid forces are applied onto the solid and the solid deformation changes the fluid domain. The kinematic conditions were 


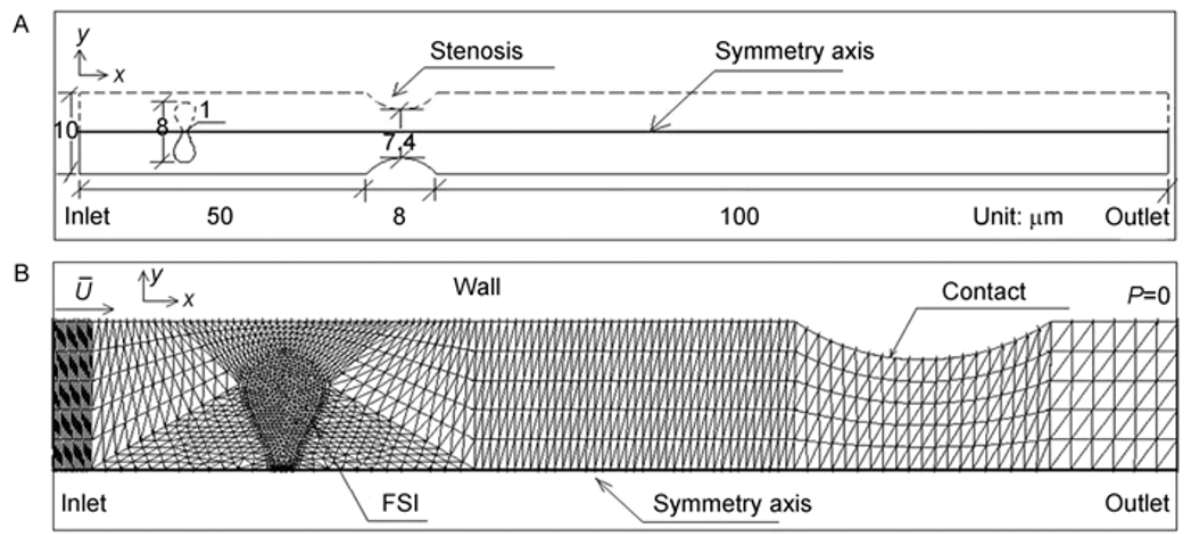

C
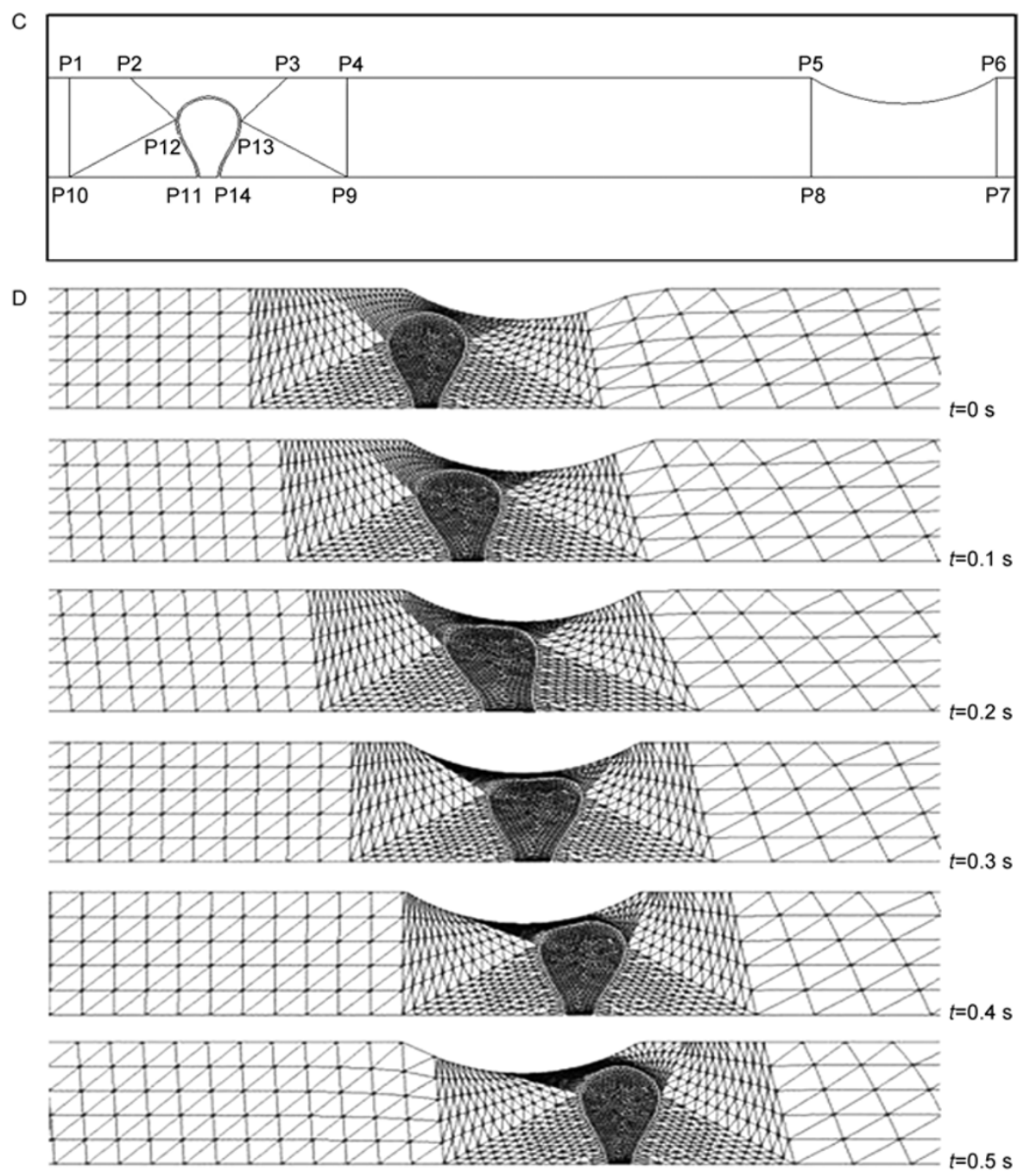

Figure 2 A schematic of the axisymmetric model used in the present work (A), showing the geometry of the microvessel and the RBC (degree of stenosis, $26 \%$ ) and the moving meshes and the boundary conditions of the computational domain (B). Only half of the microvessel was computed and 3642 nine-node axisymmetric triangular elements were used. $\bar{U}$ and FSI represent the average velocity at the inlet and the interface of the fluid-structure interaction, respectively. Points 1 to 10 are the followers and points 11 to 14 are the leaders (C). D shows the moving meshes of a single cell transiting through a microvessel stenosis. The cytoplasm-to-plasma viscosity ratio $\lambda=2.0, \beta=0.5, E=4.2 \times 10^{4} \mathrm{~Pa}$.

applied to the fluid-structure interfaces as follows: (i) The displacements of the fluid and solid domain must be compatible: $d_{\mathrm{f}}=d_{\mathrm{s}}$; (ii) the tractions at these boundaries must be at equilibrium: $n \cdot \tau_{\mathrm{f}}=n \cdot \tau_{\mathrm{s}}$; (iii) fluid must satisfy the no-slip condition: $\underline{V}=\dot{d}_{\mathrm{s}}$, where $d_{\mathrm{f}}$ and $d_{\mathrm{s}}$ are the fluid and solid displacements, $\tau_{\mathrm{f}}$ and $\tau_{\mathrm{s}}$ are the fluid and solid stresses, $n$ and $\underline{V}$ are the normal vector and the fluid velocity at the FSI interfaces, respectively. 


\subsubsection{Cell membrane model}

The membrane of the RBC was assumed to be hyperelastic, which defines a material that can completely recover its original shape following a finite deformation. Many polymers belong to this category, including cell membranes. The Mooney-Rivlin law is one of the best known and extensively used constitutive laws utilized in modeling the hyperelastic behavior of a cell membrane. The standard two-term Mooney-Rivlin constitutive law is a generalization of the Neo-Hookean solid model, where the strain energy, $W$, is a linear combination of two invariants of the Finger tensor.

$$
W=\mathrm{C}_{1}\left(\bar{I}_{1}-3\right)+\mathrm{C}_{2}\left(\bar{I}_{2}-3\right),
$$

where $\bar{I}_{1}$ and $\bar{I}_{2}$ are the first and second invariants of the deviatoric component of the Finger tensor. $\mathrm{C}_{1}$ and $\mathrm{C}_{2}$ are the material constants. If we choose $\mathrm{C}_{2}=0$, (4) will be the Neo-Hookean material law. In addition, the bulk modulus, $k$, is used to model the compressibility of the cell membrane for the plane strain and axisymmetric analysis, as follows:

$$
k=\frac{2\left(\mathrm{C}_{1}+\mathrm{C}_{2}\right)}{(1-2 v)}=\frac{E}{3(1-2 v)},
$$

where $E$ and $v$ are the Young's modulus and the Poisson's ratio of the cell membrane, respectively. The value of the Poisson's ratio is taken as 0.499 to avoid a numerical singularity, while the value of the Young's modulus ranges from $3.0 \times 10^{4} \mathrm{~Pa}$ to $5.4 \times 10^{4} \mathrm{~Pa}$, which is in the range of data obtained by previous experiments [28,29]. To investigate the effect of constants $C_{1}$ and $C_{2}$, the present study uses a new coefficient $\beta$ introduced by Li et al. [17]:

$$
\beta=\frac{\mathrm{C}_{2}}{\mathrm{C}_{1}} \text {. }
$$

The cell membrane constants $\mathrm{C}_{1}$ and $\mathrm{C}_{2}$ can then be expressed as

$$
\begin{aligned}
& \mathrm{C}_{1}=\frac{E}{6(1+\beta)}, \\
& \mathrm{C}_{2}=\frac{E \beta}{6(1+\beta)},
\end{aligned}
$$

where the range of $\beta$ extends from 0 to 0.5 .

To be more realistic, the viscoelastic effects were included in the Mooney-Rivlin material model. The equivalent one-dimensional model is shown in Figure 3. This is the same as a generalized Maxwell model with many chains. A generic chain is denoted with superscript $\alpha$, as shown in the figure. The spring $E^{\infty}$ is equivalent to the elastic stiffness of the model. Each chain contains a spring with stiffness $E^{\alpha}$ and dashpot with viscosity $\eta^{\alpha}$. The strain in each chain is the sum of the strain in the spring $g^{\alpha}$ and the strain in the dashpot $\Gamma^{\alpha}$. The observed stress is given as

$$
\sigma=\sigma^{\infty}+\sum_{\alpha} q^{\alpha}
$$

where $\sigma^{\infty}=E^{\infty} e$ is the elastic stress and $q^{\alpha}=E^{\alpha} g^{\alpha}=\eta^{\alpha} \Gamma^{\alpha}$ is the stress in chain $\alpha$. Using the definition $\tau^{\alpha}=\frac{\eta^{\alpha}}{E^{\alpha}}$ and the assumption $\beta^{\alpha}=\frac{E^{\alpha}}{E^{\infty}}$, the following expression is obtained:

$$
q^{\alpha}+\frac{1}{\tau^{\alpha}} q^{\alpha}=\beta^{\alpha} \sigma^{\infty}
$$

Considering the property of the cell membrane, the parameters $\tau^{\alpha}$ and $\beta^{\alpha}$ were set to 0.25 and 0 , respectively.

\subsubsection{Biconcave shape of RBCs}

To study the effect of the geometry (the surface-to-volume ratio) on the deformability of RBCs, three categories of blood cells were investigated, including biconcave discoid (normal), spheroidal and ellipsoidal (abnormal) cells. Evans and Fung [30] developed the following equation to describe the cross-sectional shape of the biconcave discoid:

$$
\bar{y}=0.5\left[1-\bar{x}^{2}\right]^{1 / 2}\left(C_{0}+\mathrm{C}_{1} \bar{x}^{2}+\mathrm{C}_{2} \bar{x}^{4}\right), \quad-1 \leqslant \bar{x} \leqslant 1,
$$

where $\mathrm{C}_{0}=0.207161, \mathrm{C}_{1}=2.002558, \mathrm{C}_{2}=1.122762$.

\subsection{Solution method}

In the present article, a moving mesh technology was used to simulate the flow of the RBC as it transits through the microvessel stenosis. This approach is a combination of moving meshes and leader-follower pairs. The moving mesh conditions are encountered if any boundary condition is a moving boundary condition. These conditions are given by moving walls, free surfaces, fluid-fluid interfaces and, most importantly, the fluid-structure interfaces. In the present computational model, a moving boundary condition was located on the fluid-structure interface between the cell membrane and the plasma flow, on which the displacements of nodes were determined by the structural displacements.

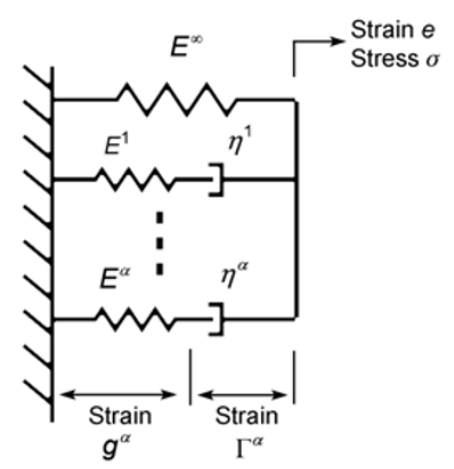

Figure 3 Equivalent one-dimensional model of rubber viscoelasticity. 
The boundary nodes could be moved along the geometric boundary as it changed position. The interior nodal movements were quite arbitrary but had to be controlled to ensure a good mesh quality. To automatically determine the displacements of those nodes that could arbitrarily be moved, we solved the Laplace equation:

$$
\nabla^{2} \Delta d=0
$$

where $\Delta d$ is the increment of the displacement. The latest displacement was then updated by adding the incremental solutions. A finite element method was applied to solve the equation based on either the latest nodal positions or the initial nodal positions.

However, Laplacian solutions cannot guarantee a valid mesh, even in some simple domains. A typical element shown in Figure 4A was distorted due to the ball movement, even though the computational domain was simple. The reason for this is that there were no moving conditions on the outer boundary of the domain. Point 2 was fixed and the line 1-2 became markedly skewed.

Because the RBC endures a large deformation in microvessel stenosis, large displacements can be encountered. To control the quality of the arbitrarily moving meshes, the leader-follower pair was used in the ALE formulation. A leader is a point located on a physically moving boundary, such as a fluid-structure interface, a moving wall or a free surface. A follower is a point whose movement is determined by its leader. The motion of the moving boundary determines the displacement of the leader. Figure 4B shows the revised situation of the distorted element in a moving boundary problem. Point 1 is the leader and point 2 is the follower. If we force point 2 to follow point 1 , then line 1-2 will not be skewed and the element maintains its quality well.

When a follower is not on the boundary of the computational domain, its motion is completely determined by its leader, as follows:

$$
\Delta d^{\mathrm{f}}=\mathrm{C} \Delta d^{1}
$$

where the superscripts indicate the leader and follower nodes and $\mathrm{C}$ is a constant (with a default value of 1.0). However, boundary followers must always stay on the boundary while they follow their leaders. Their motion is obtained from the following:

$$
\Delta d^{\mathrm{f}}=\mathrm{C} \Delta d^{1} \cdot(I-n n),
$$

where $n$ is the locally normal direction of the boundary. Figure 2C and D shows the leader-follower pairs used in the present model and the mesh deformation process of a single cell transiting through a microvessel stenosis.

\section{Results}

The rheology of the erythrocyte transiting through the microvessel with stenosis, under normal and abnormal states, was studied using the moving mesh technology combined with the finite element method. The deformation index $(D I)$ and the normalized deformation time $(T)$ were used to describe the deformability of the RBCs. The effects were studied in detail using the Reynolds number, the viscosity ratio between cytoplasm and plasma, the Mooney-Rivlin coefficients, and the surface-to-volume ratio of RBCs.

\subsection{Deformation index $(D I)$}

The deformation indices of normal (biconcave shape) and abnormal (spheroidal and ellipsoidal) RBCs are defined in Figure 5 [31].

\subsection{Normalized deformation time $(T)$}

The normalized deformation time is defined as $t / t_{\mathrm{s}}$, where $t$ and $t_{\mathrm{s}}$ are the time required for RBCs and spheroidal RBCs ( $\left.\lambda=1.0, \beta=0.5, E=4.2 \times 10^{4} \mathrm{~Pa}\right)$, respectively, to pass through the stenosis.

\subsubsection{Effect of the Reynolds number}

The effect of the Reynolds number (Re) on the deformability of RBCs was studied by changing Re from $1.5 \times 10^{-5}$ to $6.0 \times 10^{-5}$. For this set of calculations, the plasma viscosity $\mu$ was set at $1.2 \mathrm{mPa}$ s and the Young's modulus of the cell membrane $E$ was set at $4.2 \times 10^{4} \mathrm{~Pa}$. As shown in Figure 6, the maximal deformation index $(D I)_{\text {Max }}$ increased with increasing $\mathrm{Re}$, while the normalized deformation time $T$ de-

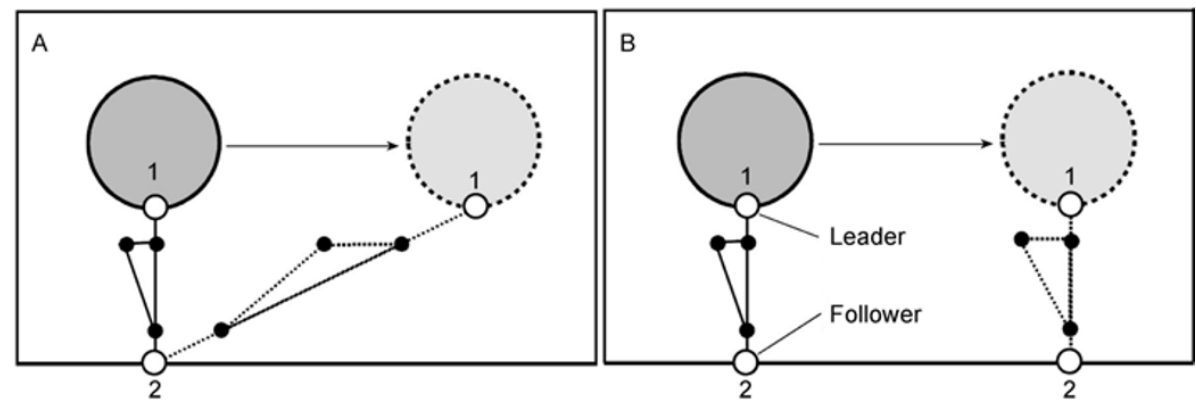

Figure 4 Elements in a moving boundary problem. A, Distorted element. B, Use of leader-follower pair. 


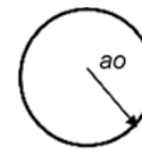

Spheroidal
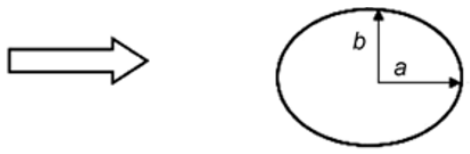

$(D I)_{\mathrm{FS}}=\frac{a-b}{a+b} \times 100 \%$
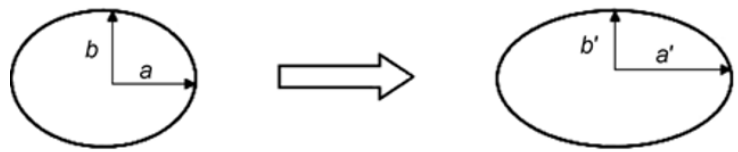

Ellipsoidal

$$
(D I)_{\mathrm{FE}}=\frac{\left(a^{\prime}-a\right)+\left(b-b^{\prime}\right)}{a^{\prime}+b^{\prime}} \times 100 \%
$$
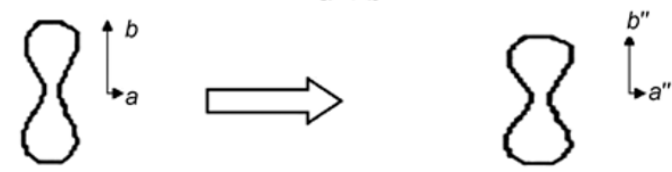

Biconcave

$$
(D I)_{\mathrm{FB}}=\frac{\left(a^{\prime \prime}-a\right)+\left(b-b^{\prime \prime}\right)}{a^{\prime \prime}+b^{\prime \prime}} \times 100 \%
$$

Figure 5 Definition of the deformation indices of three categories of RBCs, including spheroidal, ellipsoidal and biconcave shapes.

creased with increasing Re. This indicates that an increased blood flow could enhance the microcirculation, hence the oxygen and nutrients supply to human tissues and organs. The increased plasma viscosity under pathological states would lead to a decreased blood flow in microcirculation, and hence to the decreased flow rate of the RBCs.

\subsubsection{Effect of the surface-to-volume ratio}

The cell surface-to-volume ratio $\phi=S / V(S$ and $V$ are the surface area and the volume of the cell, $\mathrm{m}^{-1}$ ) is known to be a determinant of the static deformability of the normal RBC [32,33]. The present work simulated the different

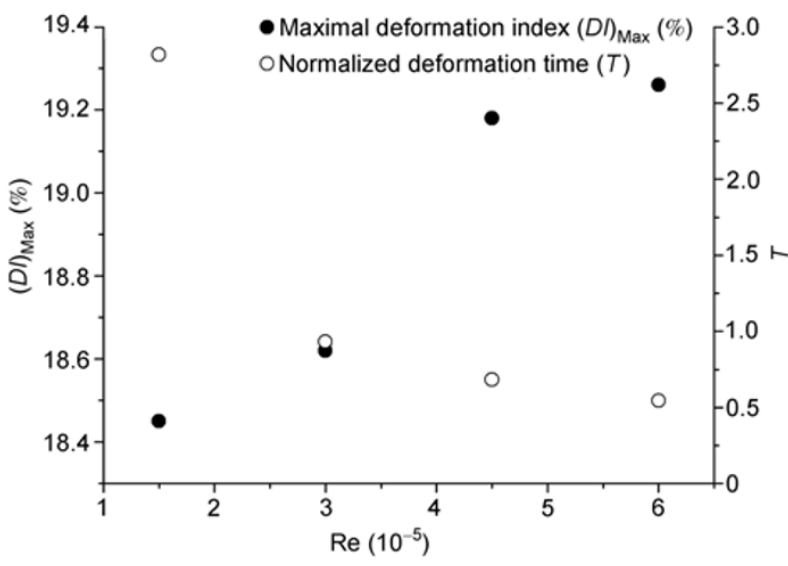

Figure 6 Distribution of the maximal deformation index $(D I)_{\operatorname{Max}}$ and the normalized deformation time $T$ under different Reynolds number Re.

shapes of RBCs flowing in a microvessel stenosis, as shown in Figure 7, which corresponded to values of $\phi$ ranging from 0.65 to 5.01. The computational results shown in Figures 8 and 9 illustrate that the maximal deformation index increased with the increasing surface-to-volume ratio, while the normalized deformation time decreased with the increasing surface-to-volume ratio. This may imply that the biconcave discoid shape is the optimal shape for RBCs and that the spheroidal or ellipsoidal shape is detrimental to the transit of RBCs through microvessel stenoses.

\subsubsection{Effect of the Mooney-Rivlin coefficients}

To investigate the effect of cell membrane properties on the deformability of RBCs, a series of simulations was carried out using various Mooney-Rivlin coefficients, such as the Young's modulus $E$ and the new coefficient $\beta$. Figure 10 illustrates the effect of Young's modulus by changing $E$

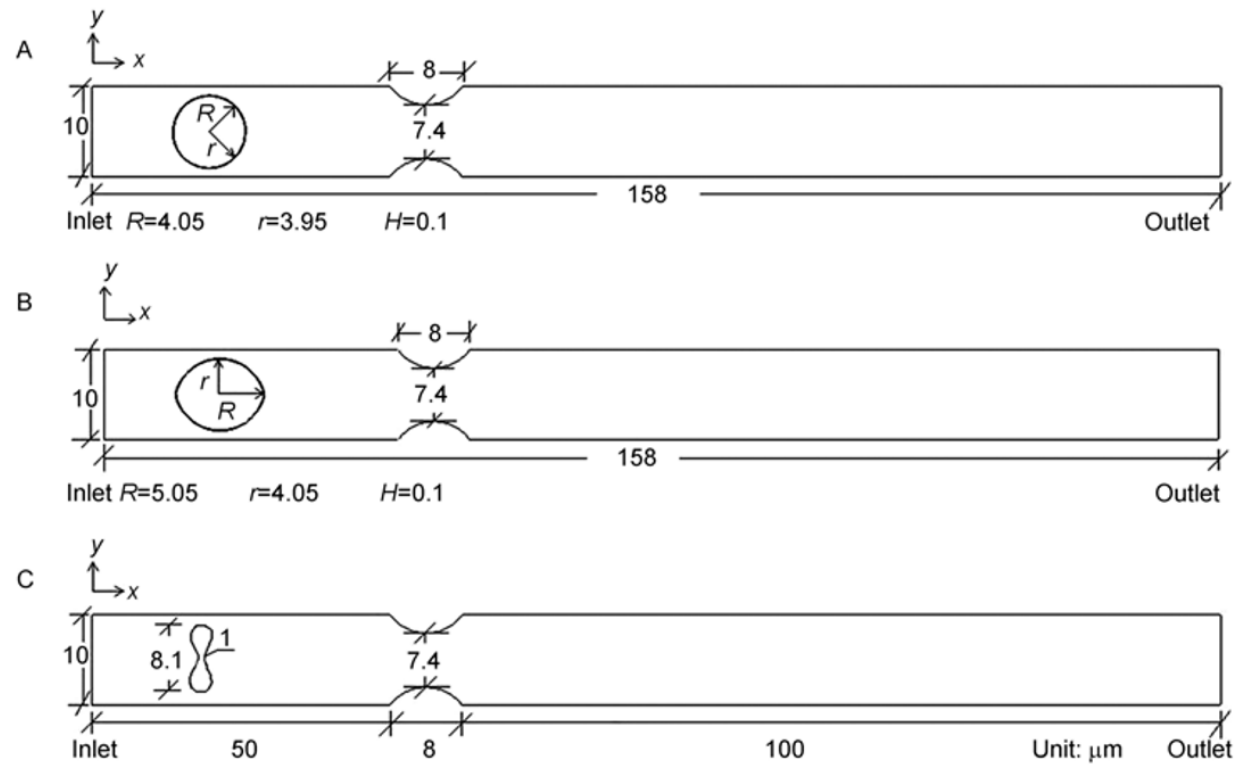

Figure 7 A schematic of spheroidal, ellipsoidal, and biconcave cells flowing in a microvessel stenosis. 


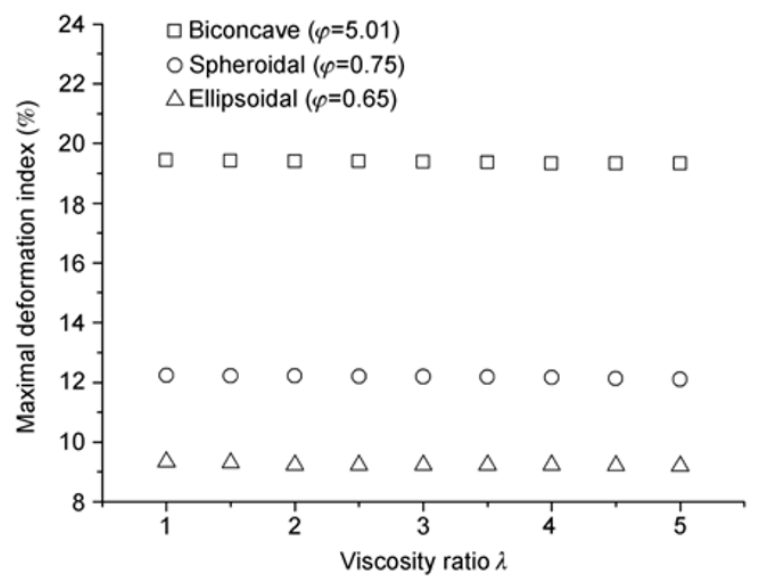

Figure 8 Distribution of the maximal deformation index $(D I)_{\text {Max }}$ along the cytoplasm-to-plasma viscosity ratio $\lambda$ under different surface-tovolume ratios $\phi$.

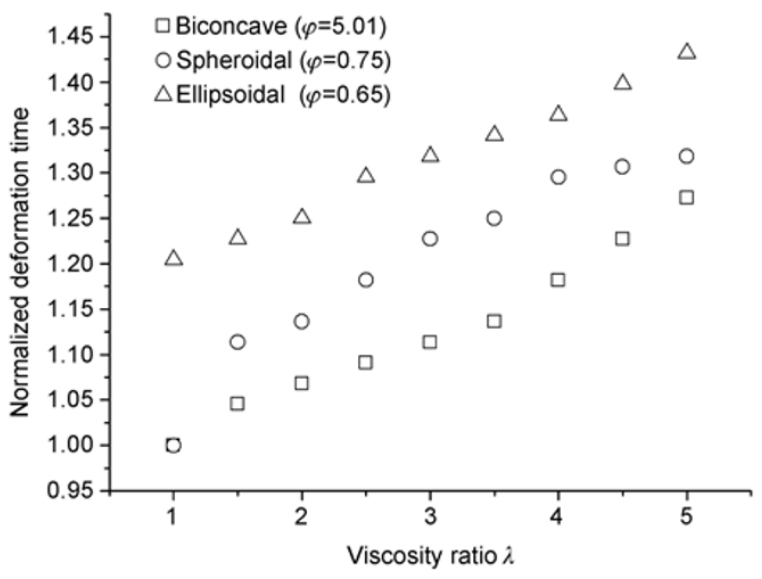

Figure 9 Distribution of the normalized deformation time $T$ as a function of the cytoplasm-to-plasma viscosity ratio $\lambda$ under different surface-tovolume ratios $\phi$.

from $2.4 \times 10^{4} \mathrm{~Pa}$ to $5.4 \times 10^{4} \mathrm{~Pa}, \beta=0.5$. As shown in the figure, the higher the value of the $E$, the lower the value of $(D I)_{\text {Max }}$ and the longer the time required for the cell to pass through the stenosis. Figure 11 summarizes the effect of $\beta$ by changing it from 0.1 to $0.5, E=4.2 \times 10^{4} \mathrm{~Pa}$. As is evident in the figure, the higher the value of $\beta$, the higher the value of $(D I)_{\text {Max }}$ and the shorter the time required for the cell to pass through the stenosis.

\subsubsection{Effect of the viscosity ratio between cytoplasm and plasma}

A series of simulations under different cytoplasm-to-plasma viscosity ratios was carried out. The viscosity ratio was given by $\lambda=\mu_{\mathrm{c}} / \mu_{\mathrm{p}}$, where $\mu_{\mathrm{c}}$ and $\mu_{\mathrm{p}}$ are the viscosities of the cytoplasm and the plasma, respectively. The majority of previous reports in the literature have shown that cells suspended in a high-viscosity Dextran or buffer suspending medium have a low viscosity ratio, always less than 1.0,

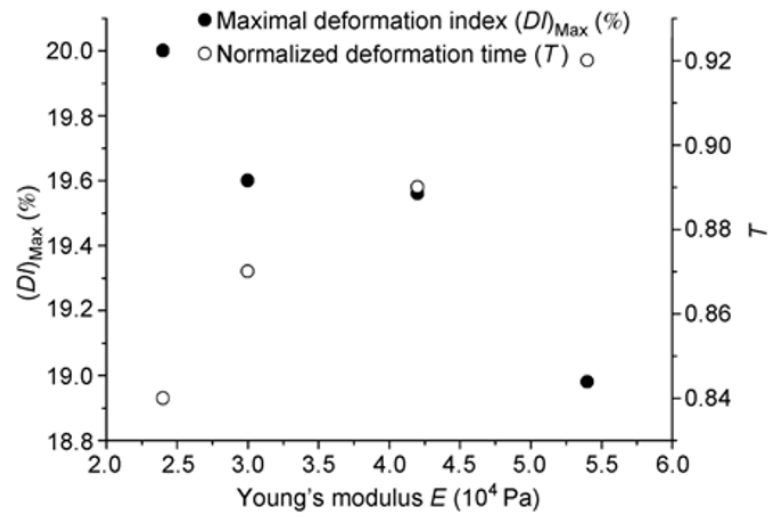

Figure 10 Relationship between the maximal deformation index $(D I)_{\mathrm{Max}}$ and the normalized deformation time $T$ under different Young's modulus $E$.

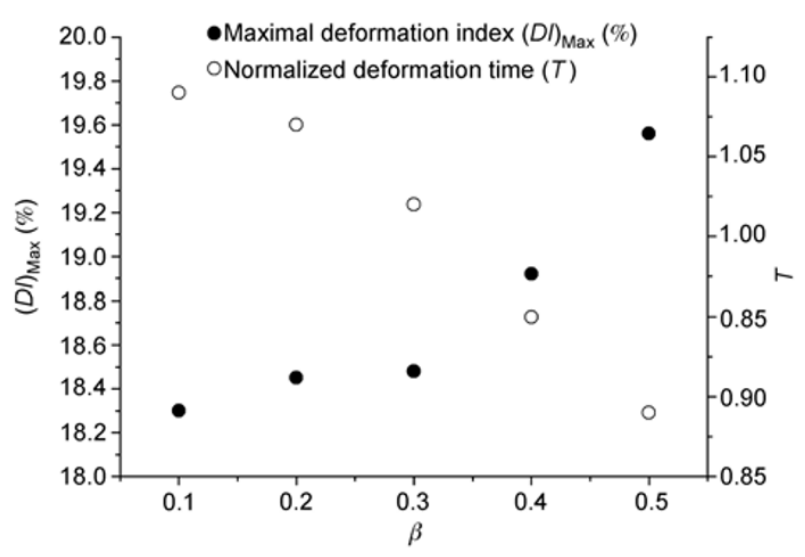

Figure 11 Relationship between the maximal deformation index $(D I)_{\text {Max }}$ and the normalized deformation time $T$ under different material coefficients $\beta$.

while the viscosity ratio of RBCs suspended in plasma always ranges between 5.0 and $10.0[13,34,35]$. In this set of simulations, the viscosity of the cytoplasm was set at 6.0 $\mathrm{mPa} \mathrm{s}$ and that of the plasma was in the range between 1.2 and $6.0 \mathrm{mPa}$ s, corresponding to a viscosity ratio range from 1.0 to 5.0, which covers both experimental and physiological fields [36]. As shown in Figure 12, the value of the $(D I)_{\text {Max }}$ decreased with increasing values of $\lambda$, while $T$ increased with increasing $\lambda$.

\section{Conclusion}

In the human blood circulatory system, macrovascular tissues represent only a small fraction of the system, while microvessels with diameters of the same size as RBCs number about one billion. In these capillaries, the rheology of the RBC is an extremely important determinant of the overall blood flow, as even a slight decrease in the rheology of RBC can result in a sharp decrease in microvessel flow rate. The decreased fluidity of the microvessel flow will in turn lead to a reduced microcirculatory flow and conse- 


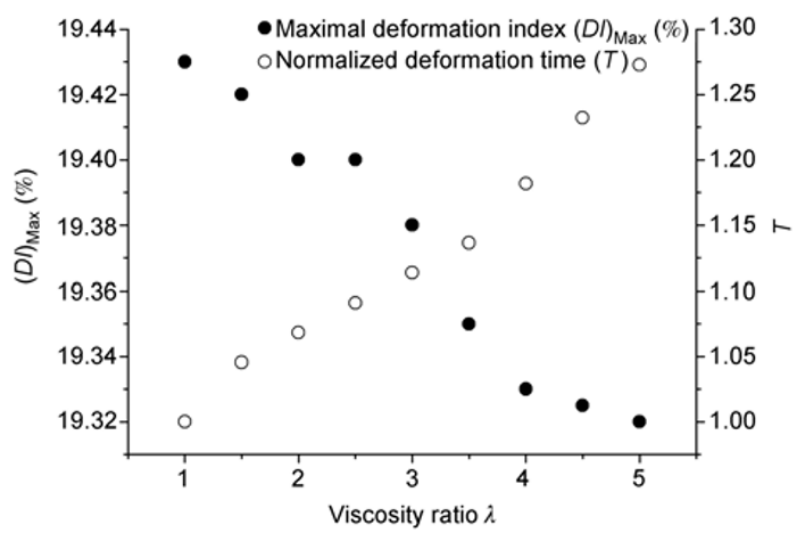

Figure 12 Relationship between the maximal deformation index $(D I)_{\text {Max }}$ and the normalized deformation time $T$ under different cytoplasm-to-plasma viscosity ratios $\lambda$.

quently, reduced oxygen supply to the tissues. These defects are very dangerous if they take place in vital parts of the human body, particularly in the brain. In the falciparum malaria study of Dondorp et al. [37], logistic regression analysis showed that RBC deformability was the single most important prognostic factor for mortality, more important than other parameters, such as plasma lactate concentrations and other measures of acid/base status.

The present study used a moving mesh technology that incorporated leader-follower pairs to simulate the mechanical behavior of a single RBC in a microvessel with stenosis, to investigate the relationship between cell deformability and relative parameters such as the surface-to-volume ratio of the erythrocyte, the viscosity ratio between cytoplasm and plasma, the Reynolds number of the blood flow and the material coefficients of the cell membrane. Although it is a simplified model of actual human microcirculation, which involves more complex motions of leucocytes and multiple RBCs, it included all the pertinent problems of concern. With the further development of such numerical methods, the direct numerical simulation of realistic flow in human microvessels is expected to be attained in the near future.

When the whole-blood viscosity is raised, for example by diseases such as malaria and diabetic nephropathy, flow rate may decrease; hence, the fluidity of the RBCs flow also decreases, which is consistent with our numerical simulation (Figure 6). Computational results show that under a constant flow rate, the transit time of a RBC passing through a stenosis clearly decreased with increasing plasma viscosity (Figure 12). This differs from the result obtained by Secomb et al. [38], who postulated that an elevation in suspending medium viscosity would not increase the sensitivity of transit time to membrane properties. For these reasons, we believe that decreased hemorheology in microvessels under pathological states may be attributed primarily to an increase in the numbers of white blood cells, which will result in the aggregation of RBCs and a change in the blood flow pattern.
The results of the present study also support the hypothesis that the accumulation of sickle RBCs formed in a microvessel stenosis will result in the aggregation and cytoadherence of RBCs. This would decelerate the microcirculation flow and contribute to microvascular obstruction. Furthermore, we believe that a lower flow rate will give rise to a smaller wall shear stress. By contrast, a longer time deposition of atherogenic lipids onto the microvessel wall would lead to the formation of a thrombosis. It is well known that thrombosis is an important defect in the blood circulatory system and is a major cause of most heart attacks, strokes, and other severe cardiovascular problems [36]. Obviously, drugs aimed at improving the rheology of RBCs in a microvessel stenosis are a promising area for future medical research.

This work was supported by the National Natural Science Foundation of China (Grant No. 10672090) and the National High Technology Research and Development Program of China (Grant No. 2006AA02Z4E8). Software was provided by the Adina Company, USA.

1 Secomb T W, Hsu R, Pries A R. A model for red blood cell motion in glycocalyx-lined capillaries. Am J Physiol Heart Circ Physiol, 1998, 274: 1016-1022

2 Secomb T W, Hsu R, Pries A R. Motion of red blood cells in a capillary with an endothelial surface layer: Effect of flow velocity. Am J Physiol Heart Circ Physiol, 2001, 281: 629-636

3 Dong C, Lei X X. Biomechanics of cell rolling: Shear flow, cellsurface adhesion, and cell deformability. J Biomech, 2000, 33: 35-43

4 Lei X, Lawrence M R, Dong C. Influence of cell deformation on leucocyte rolling adhesion in shear flow. J Biomech Eng, 1999, 121: 636-643

5 Evans E A. New membrane concept applied to the analysis of fluid shear and micropipette-deformed red blood cells. Biophys J, 1973, 13: 941-954

6 Henon S, Lenormand G, Richert A. A new determination of the shear modulus of the human erythrocyte membrane using optical tweezers. Biophys J, 1999, 76: 1145-1151

7 Bronkhorst P J H, Streekstra G J, Grimbergen J. A new method to study shape recovery of red blood cells using multiple optical trapping. Biophys J, 1995, 69: 1666-1673

8 Engelhardt H, Sackmann E. On the measurement of shear elastic moduli and viscosities of erythrocyte plasma membranes by transient deformation in high frequency electric fields. Biophys J, 1988, 54: 495-508

9 Fung Y C. Stochastic flow in capillary blood vessels. Microvasc Res, 1973, 5: 34-49

10 Keller S R, Skalak R. Motion of a tank-treading ellipsoidal particle in a shear flow. J Fluid Mech, 1982, 120: 27-47

11 Kennedy M, Pozrikidis C, Skalak R, et al. Motion and deformation of liquid drops, and the rheology of dilute emulsions in shear flow. Comput Fluids, 1994, 23: 251-278

12 Zhou H, Pozrikidis C. Deformation of liquid capsules with incompressible interfaces in simple shear flow. J Fluid Mech, 1995, 283: 175-200

13 Ramanujan S, Pozrikidis C. Deformation of liquid capsules enclosed by elastic membranes in simple shear flow: Large deformations and the effect of capsule viscosity. J Fluid Mech, 1998, 361: 117-143

14 Cristini V, Blawzdziewicz J, Loewenberg M. An adaptive mesh algorithm for evolving surfaces: Simulations of drop breakup and coalescence. J Comput Phys, 2001, 168: 445-463

15 Pozrikidis C. Numerical simulation of the flow-induced deformation 
of red blood cells. Ann Biomed Eng, 2003, 31: 1194-1205

16 Sugihara M, Niimi H. Numerical approach to the motion of a red blood cell in Couette flow. Biorheology, 1984, 21: 735-749

17 Li C, Liu Y P, Liu K K. A parameter study of the material properties in the vesicle-fluid interaction. Comput Mater Sci, 2004, 30: 504-510

18 Chee C Y, Lee H P, Lu C. Using 3D fluid-structure interaction model to analyse the biomechanical properties of erythrocyte. Phys Lett A, 2008, 372: 1357-1362

19 Eggleton C D, Popel A S. Large deformation of red blood cell ghosts in a simple shear flow. Phys Fluids, 1998, 10: 1834-1845

20 Prosenjit B. Mesoscale simulation of blood flow in small vessels. Biophys J, 2007, 92: 1858-1877

21 Barthes-Biesel D. Motion of a spherical microcapsule freely suspended in a linear shear flow. J Fluid Mech, 1980, 100: 831-853

22 Barthes-Biesel D, Rallison J M. The time-dependent deformation of a capsule freely suspended in a linear shear flow. J Fluid Mech, 1981, 113: 251-267

23 Toru H, Shouko T, Takeshi M. Numerical study on flows of red blood cells with liposome-encapsulated hemoglobin at microvascular bifurcation. J Biomech Eng, 2008, 130: 110141-110146

24 Weed R I. The importance of erythrocyte deformability. Am J Med, 1970, 49: 147-150

25 Chien S. Haemorheology in disease: Pathophysiological significance and therapeutic implications. Clin Hemorheol, 1981, 1: 419-442

26 Chien S. Red cell deformability and its relevance to blood flow. Ann Rev Physiol, 1987, 49: 177-192

27 Liu Q, Mirc D, Fu B. Mechanical mechanisms for thrombosis in microvessels. In: Engineering in Medicine and Biology Society, Annual International Conference of the IEEE, New York, USA, 2001. 173-174

28 Rotsch C, Jacobson K, Radmacher M. Dimensional and mechanical dynamics of active and stable edges in motile fibroblasts investigated by using atomic force microscopy. Proc Natl Acad Sci USA, 1999, 3: 921-926

29 Hofmann U G, Rotsch C, Parak W J. Investigating the cytoskeleton of chicken cardiocytes with the atomic force microscope. J Struct Biol, 1997, 119: 84-91

30 Evans E, Fung Y C. Improved measurements of the erythrocyte geometry. Microvasc Res, 1972, 4: 335-347

31 Liu X, Tang Z Y, Zeng Z, et al. The measurement of shear modulus and membrane surface viscosity of RBC membrane with Ektacytometry: A new technique. Math Biosci, 2007, 209: 190-204

32 Linderkamp, Meiselman H J. Geometric, osmotic and membrane mechanical properties of density-separated human red cells. Blood, 1982, 59: 1121-1127

33 Mohandas N, Clark M R, Jacobs M S. Analysis of factors regulating erythrocyte deformability. J Clin Invest, 1980, 66: 563-573

34 Fischer T, Stohr-Liesen M, Schmid-Schonbein H. The red cell as a fluid droplet: Tank tread-like motion of the human erythrocyte membrane in shear flow. Science, 1978, 202: 894-896

35 Schmid-Schonbein H, Wells R. Fluid drop-like transition of erythrocytes under shear. Science, 1969, 165: 288-291

36 Zhang Z G, Deng X Y, Fan Y B. The effects of recirculation flows on mass transfer from the arterial wall to flowing blood. ASAIO J, 2008, 54: $37-43$

37 Dondorp A M. Prognostic significance of reduced RBC deformability in severe falciparum malaria. Am J Trop Med Hyg, 1997, 57: 507-511

38 Secomb T W, Hsu R. Analysis of red blood cell motion through cylindrical micropores: Effects of cell properties. Biophys J, 1996, 71: 1095-1101

Open Access This article is distributed under the terms of the Creative Commons Attribution License which permits any use, distribution, and reproduction in any medium, provided the original author(s) and source are credited. 